\title{
AUTOMORPHIC FORMS AND SINGULARITIES OF COMPLEX SURFACES
}

\author{
BY PHILIP WAGREICH ${ }^{1}$
}

DEDICATED TO MY WIFE, BOBBIE

Suppose $G$ is a finitely generated fuchsian group of the first kind. Let $A(k)$ be the vector space of entire automorphic forms of weight $k$ and

$$
A(G)=\bigoplus_{k \geqslant 0} A(k)
$$

the graded ring of automorphic forms. Now $G$ acts on the upper half plane $H_{+}$in the usual way. This action has a 'canonical' extension to $H_{+} \times \mathbf{C} *$ via

$$
g(z, t)=\left(g(z), \frac{d g}{d z} t\right) .
$$

PROPOSITION 1. $A(G)$ is a graded algebra of finite type. The algebraic set $V=\operatorname{Spec}(A(G))$ is a surface with $\mathbf{C}^{*}$-action. There is a Zariski open $\mathbf{C}^{*}$-invariant subset of $V$ which is isomorphic to $\left(H_{+} \times \mathrm{C}^{*}\right) / G$.

We thus can use the theory of surfaces with $\mathrm{C}^{*}$-action to study the structure of the ring of automorphic forms. Now let us suppose that $G$ is a fuchsian group with signature $\langle g ; s ; e(1), \ldots, e(r)\rangle$ and $V=\operatorname{Spec}(A(G))$. By [7] the singularity of $V$ at ( 0$)$ has a canonical equivariant resolution. The graph of the resolution is star shaped, of the form

where $b=2 g-2+r+s$.

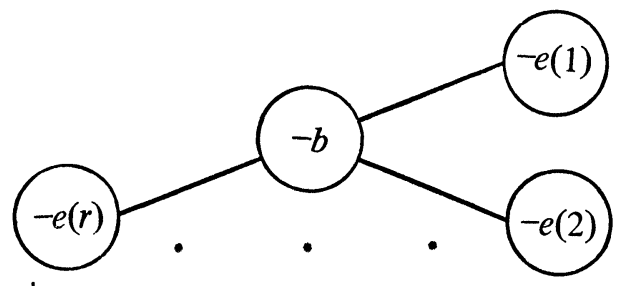

A first step in understanding the structure of these rings is to find the minimal number of generators, $n$. In [9] we classified all groups with $n \leqslant 3$. The techniques there are all elementary. The results here are more general since we use

Received by the editors October 30, 1978.

AMS (MOS) subject classifications (1970). Primary 30A58; Secondary 14J15.

Key words and phrases. Automorphic form, graded ring, surface with $\mathrm{C}^{*}$-action, singularity with $\mathrm{C}^{*}$-action, Fuchsian group.

${ }^{1}$ Research partially supported by grants from the National Science Foundation. 
the more powerful techniques from the theory of singularities of surfaces. The groups with $n \leqslant 3$ and $s=0$ were also classified by Dolgacev [4]. If $g=0$ and $s>0$ then the singularity is a rational singularity and one can use the theory of these singularities to compute $n$. First we let $e=\Sigma_{i=1}^{r} e(i)$.

Proposition 2. (1) If $s>1$ then $n=e-r+s-1$,

(2) If $s=1$ then $n=e-3$.

If $g=0$ and $s=0$ then the singularity is minimal elliptic [6], hence it follows directly that:

Proposition 3. (1) if $r>3$ then $n=\max (3, e-8)$,

(2) If $r=3$ and $e(i)>2$, for all $i$, then

$$
n=\max (3, e-9) \text {. }
$$

(3) If $r=3, e(1)=2, e(2), e(3)>3$ then

$$
n=\max (3, e-10) \text {. }
$$

(4) If $r=3, e(1)=2, e(2)=3, e(3)>6$ then

$$
n=\max (3, e-11) \text {. }
$$

To get more information about generators one can use the following description of $A(k)$ as a vector space of functions on $X$.

Proposition 4 [8], [9]. Suppose that $p(1), \ldots, p(r) \in X$ are the elliptic points and $q(1), \ldots, q(s) \in X$ are the cusps. Then

$$
A(k)=L\left(k K+k\left(q((1)+\cdots+q(s))+\sum_{i=1}^{r}[k(1-1 / e(i))] p(i)\right.\right.
$$

A major tool for stating and proving our results is the Poincare power series of the graded algebra $A(G)$. Recall that if $R$ is any graded algebra over a field $K$ and $M$ is a finitely generated $R$-module, then the Poincaré power series of $M$ is defined to be

$$
p(t)=\sum_{i=0}^{\infty} d(i) t^{i}
$$

where $d(i)=$ dimension of $R(i)$ as a vector space over $K$. Moreover, if $R$ is finitely generated as an algebra over $K$ then $p(t)$ is a rational function [2]. Now let $m$ be the maximal ideal of $A(G)$ defined by

$$
m=\bigoplus_{k>0} A(k) .
$$


Then any basis of $\mathrm{m} / \mathrm{m}^{2}$ as a vector space over $\mathbf{C}$ lifts to a minimal set of generators of the algebra $A(G)$. Conversely, every minimal set of algebra generators forms a basis for $m / m^{2}$. Now $m$ is a graded ideal, hence there is an induced grading on $m / \mathrm{m}^{2}$. Let $p(t)$ be the Poincare power series of $\mathrm{m} / \mathrm{m}^{2}$. Of course this is just a polynomial. The coefficient of $t^{i}$ in this polynomial is just the number of independent generators of weight $i$.

THEOREM. If $n>3$ then

$$
p(t)=f(t)+\sum_{i=1}^{r}\left(t^{2}+\cdots+t^{e(i)}\right)
$$

where $f(t)$ is given in the table below.

\section{SIGNATURE}

$s \geqslant 3$ or $g=0$ and $s=2$

$f(t)$

$s=2, g \geqslant 2$ and $1(q(1)+q(2))=1$

$(g+s-1) t$

$s=2, g \geqslant 1$ and $1(q(1)+q(2))=2$

$(g+1) t+t^{2}$

$s=1, g \geqslant 3, X$ nonhyperelliptic

$(g+1) t+g t^{2}$

$s=1, g \geqslant 1, X$ hyperelliptic

$g t+2 t^{2}+t^{3}$

$s=1, g=0, r \geqslant 2$

$g t+g t^{2}+t^{3}$

$s=0, g \geqslant 3, X$ nonhyperelliptic

$-t^{2}+(r-2) t^{3}$

$s=0, g \geqslant 2, X$ hyperelliptic, $g+r \geqslant 3$

$g t$

$s=0, g=1$ and either $e \geqslant 6$ or $r=1, e(1) \geqslant 4$

$g t+(g-2) t^{2}$

$t-t^{2}$

$s=0, g=0, r \geqslant 4, e \geqslant 11$

$-3 t^{2}+(r-5) t^{3}$

$s=0, g=0, r=3, e(i) \geqslant 3$, for all $i$.

$-3 t^{2}-2 t^{3}-t^{4}$

$s=0, g=0, r=3, e(1)=2, e(2), e(3) \geqslant 4, e \geqslant 13$

$-3 t^{2}-2 t^{3}-t^{4}-t^{5}$

$s=0, g=0, r=3, e(1)=2, e(2)=3, e(3) \geqslant 9$

$-3 t^{2}-2 t^{3}-t^{4}-t^{5}-t^{7}$

The finite number of signatures which do not appear above all have $A$ generated by 2 or 3 elements. The generators and relations for these rings are listed in [9].

COROLlary. If we are as above, then $n=e-r+f(1)$.

\section{EMBEDDING DIMENSION 4}

The following is a list of all groups whose algebra of automorphic forms is generated by four elements. If the algebra is a complete intersection, that is the ideal of relations is generated by two elements, then we give the degree of the generating relations in the last column. 


\section{SIGNATURE}

$\langle 4\rangle, X$ nonhyperelliptic

$\langle 3\rangle, X$ hyperelliptic

$\langle 3 ; 0 ; 2\rangle, X$ nonhyperelliptic

$\langle 2 ; 0 ; 2,2\rangle$

$\langle 2 ; 0 ; 3\rangle$

$\langle 2 ; 2\rangle q(1)+q(2)$ not linearly equivalent to $K$.

$\langle 2 ; 3\rangle$

$\langle 1 ; 0 ; 2,2,2,2\rangle$

$\langle 1 ; 0 ; 2,2,3\rangle$

$\langle 1 ; 0 ; 2,4\rangle$

$\langle 1 ; 0 ; 3,3\rangle$

$\langle 1 ; 0 ; 5\rangle$

$\langle 1 ; 1 ; 2\rangle$

$\langle 1 ; 2 ; 2\rangle$

$\langle 1 ; 3 ; 2\rangle$

$\langle 1 ; 4\rangle$
DEGREES OF GENERATORS RELATIONS

$\begin{array}{llll}1 & 1 & 1 & 1\end{array}$

23

1112

24

1112

33

1122

34

1123

44

1112

334

1111

233

1222

44

1223

45

1234

56

1233

46

1345

68

1223

456

1122

344

1112

333

22

The groups with $g=0$ are easily found using Propositions 1 and 2 . There are 25 signatures that occur in this case.

\section{BIBLIOGRAPHY}

1. M. Artin, On isolated rational singularities of surfaces, Amer. J. Math. 88 (1966), 129-136.

2. M. Atiyah and I. MacDonald, Introduction to commutative algebra, AddisonWesley, Reading, Mass., 1969.

3. P. Connor and F. Raymond, Holomorphic seifert fiberings, Proceedings of the Second Conference on Compact Transformation Groups, Lecture Notes in Math., vol. 299, Springer-Verlag, Berlin and New York.

4. I. Dolgacev, Automorphic forms and quasi-homogeneous singularities, Functional Anal. Appl. 9 (1975), 149-151.

5. - Automorphic forms and quasi-homogeneous singularities (preprint).

6. H. Laufer, On minimally elliptic singularities, Amer. J. Math. 99 (1977), 12571295.

7. P. Orlik and $\mathrm{P}$. Wagreich, Isolated singularities of surfaces with $\mathrm{C}^{*}$-action, Ann. of Math. (2) 93 (1971), 205-228. 183-193.

8. H. Pinkham, Normal surface singularities with $\mathrm{C}^{*}$-action, Math. Ann. 227 (1977),

9. P. Wagreich, Algebras of automorphic forms with few generators (preprint).

DEPARTMENT OF MATHEMATICS, UNIVERSITY OF ILLINOIS AT CHICAGO CIRCLE, CHICAGO, ILLINOIS 60680 